Document downloaded from:

http://hdl.handle.net/10251/69717

This paper must be cited as:

Aguilar, A.; González Martínez, AJ.; Torres, J.; García Olcina, R.; Martos, J.; Soret, J.; Conde Castellanos, PE.... (2015). Timing results using an FPGA-based TDC with large arrays of 144 SiPMs. IEEE Transactions on Nuclear Science. 62(1):12-18. doi:10.1109/TNS.2014.2359078.

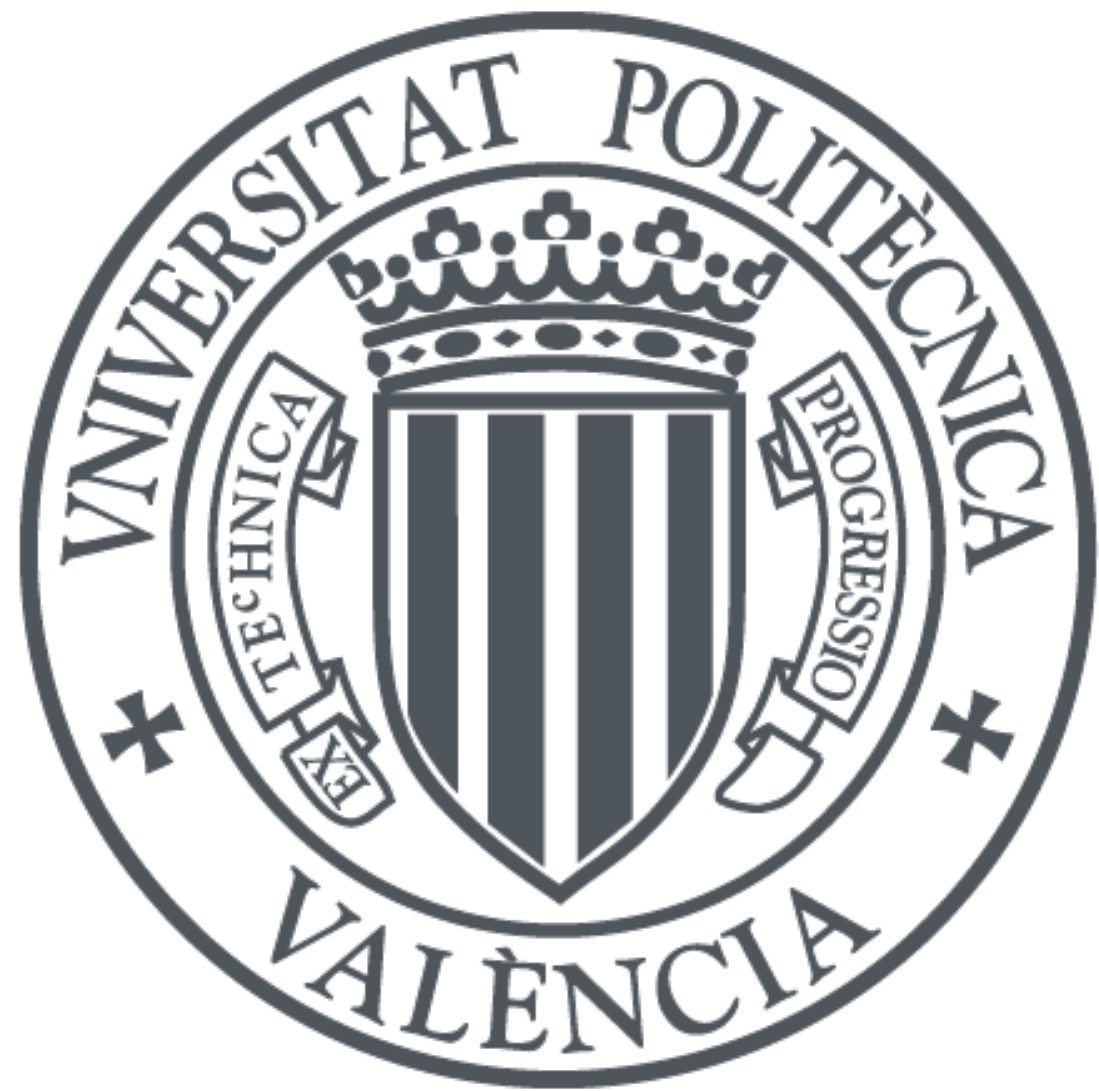

The final publication is available at

http://dx.doi.org/10.1109/TNS.2014.2359078

Copyright Institute of Electrical and Electronics Engineers (IEEE)

Additional Information 


\title{
Timing Results Using an FPGA-Based TDC with Large Arrays of 144 SiPMs
}

\author{
A. Aguilar, A. J. González, J. Torres, R. García-Olcina, J. Martos, J. Soret, P. Conde, L. Hernández, \\ F. Sánchez, and J. M. Benlloch
}

\begin{abstract}
Silicon photomultipliers (SiPMs) have become an alternative to traditional tubes due to several features. However, their implementation to form large arrays is still a challenge especially due to their relatively high intrinsic noise, depending on the chosen readout. In this contribution, two modules composed of $12 \times 12 \mathrm{SiPMs}$ with an area of roughly $50 \mathrm{~mm} \times 50 \mathrm{~mm}$ are used in coincidence. Coincidence resolving time (CRT) results with a field-programmable gate array, in combination with a time to digital converter, are shown as a function of both the sensor bias voltage and the digitizer threshold. The dependence of the CRT on the sensor matrix temperature, the amount of SiPM active area and the crystal type is also analyzed. Measurements carried out with a crystal array of $2 \mathrm{~mm}$ pixel size and $10 \mathrm{~mm}$ height have shown time resolutions for the entire $288 \mathrm{SiPM}$ two-detector set-up as good as 800 ps full width at half maximum (FWHM).
\end{abstract}

Index Terms-Nuclear medicine, positron emission tomography (PET), silicon radiation detectors, trigger circuits.

\section{INTRODUCTION}

M OST conventional Positron Emission Tomography (PET) systems are only capable of determining the line of response connecting the two-photon impacts registered within a coincidence window. But there is further information that can be used during the image reconstruction process. Adding the so-called Time of Flight (TOF) information to such systems allows one to measure the arrival time difference of the two gamma rays, providing a better localization of the annihilation event along the coincidence line. Nevertheless, even after enabling the TOF capability, the spatial position is subject to a certain time uncertainty named the Coincidence Resolving Time (CRT). Despite this uncertainty, it is possible to use such a method to reduce the coincidence window and, thus, to reduce the influence of false coincidences, which decreases the noise in the final image.

The TOF benefits for PET systems have been amply demonstrated. Amongst them, the fastest convergence of the reconstruction algorithm and a reduction in the tracer dose for the

Manuscript received February 21, 2014; revised July 07, 2014; accepted September 15,2014 . This work was supported in part by the University of Valencia and the Institute for Instrumentation and Molecular Imaging.

A. Aguilar, J. Torres, R. García-Olicna, J. Martos, and J. Soret are with the Communications and Digital Systems Design group (DSDC), Universidad de Valencia, Valencia, 46010, Spain (e-mail: albert.aguilar@uv.es).

A. J. González, J. M. Benlloch, P. Conde, L. Hernández, and F. Sánchez are with the Institute for Instrumentation in Molecular Imaging (I3M), Universidad Politécnica de Valencia-CSIC-CIEMAT, Valencia, 46022, Spain.

Color versions of one or more of the figures in this paper are available online at http://ieeexplore.ieee.org. patient have been pointed out [1]. Due to the limitations of scintillators and photodetectors that are currently feasible for use in a commercial PET scanner, relatively few PET systems implementing this capability currently exist [2].

A new technique for fine time measurements has appeared using field-programmable gate arrays (FPGAs) [3]-[6]. Taking advantage of the FPGA capabilities, the authors have developed a multichannel Time-to-Digital Converter (TDC) core, and CRT values below $100 \mathrm{ps}$ full width at half maximum (FWHM) have been obtained when tested with a pulse generator for 24 simultaneous input channels [6]. In this work, performance experiments using such an FPGA-based TDC will be shown when SiPM arrays are used as photosensors.

Most available PET systems use position sensitive photomultiplier tubes as photosensor detectors, but there is a trend to replace them with solid-state photosensors, such as silicon photomultiplier (SiPM) detectors [7]-[10]. SiPMs are based on avalanche photodiodes connected in a matrix and working in the Geiger regime. This mode of operation allows the device to work slightly above its breakdown voltage, which induces exponential avalanches allowing work at high gain rates. Very recently, an alternative to such analog SiPMs has appeared, the so-called digital SiPMs [11].

There are several features that have strongly contributed to the photomultiplier tube's (PMTs) replacement by SiPMs. The operation voltage of typical PMTs is well above $700 \mathrm{~V}$ whereas SiPMs are able to operate below $100 \mathrm{~V}$ (some models below $50 \mathrm{~V}$ ). Furthermore, SiPMs present better quantum efficiency, i.e., the number of impact photons that generate an electrical current is higher $(50 \%$ compared to $25 \%$ in the case of most PMTs). Parameters that limit time resolution such as transit time spread (time jitter) are improved in SiPMs, making their use feasible for TOF measurements [12]-[14]. Moreover, they are able to work in magnetic fields whereas PMTs are highly sensitive to such environments. This feature has been decisive in including SiPMs in new PET systems for working in combination with Magnetic Resonance Imaging (MRI). Conversely, SiPMs are temperature dependent sensors [5]. Therefore, they must be carefully controlled when accurate time resolution measurements are targeted.

In the last few years, different studies based on SiPMs applied to PET systems have been carried out (for more details, see Table I). In [16], two SiPMs coupled to LSO crystals were used in coincidence and a CRT of $1.4 \mathrm{~ns}$ FWHM was obtained. In [17] a LSO crystal array and a $2 \times 2 \mathrm{SiPM}$ array was also tested, obtaining 1.9 ns FWHM CRT. The authors of [18] investigated the operation of SiPMs inside a 3T MRI resulting on 1.3 ns FWHM CRT with LYSO pixels. All these results exhibit time resolutions above $1 \mathrm{~ns}$, which prevents useful TOF 
TABLE I

State of the ART in SiPM Detector ARrays AND their Associated CRT

\begin{tabular}{lccc}
\hline \hline Ref. & $\begin{array}{c}\text { Crystal pixel size } \\
\left(\mathbf{m m}^{3}\right) / \text { array overall } \\
\text { size }\left(\mathbf{m m}^{3}\right)\end{array}$ & $\begin{array}{c}\text { SiPM's size } \\
\left(\mathbf{m m}^{2}\right) / \text { array overall } \\
\text { size }\left(\mathbf{m m}^{2}\right)\end{array}$ & $\begin{array}{c}\text { CRT } \\
\text { FWHM } \\
(\mathbf{p s})\end{array}$ \\
\hline \hline $16^{\mathrm{A}}$ & $1 \times 1 \times 10 / 1 \times 1 \times 10$ & $1 \times 1 / 1 \times 1$ & 1400 \\
$17^{\mathrm{A}}$ & $1.4 \times 1.4 \times 12 / 1.4 \times 1.4 \times 12$ & $1 \times 1 / 1 \times 1$ & 1900 \\
18 & $2 \times 2 \times 10 / 2 \times 2 \times 10$ & $2 \times 2 / 2 \times 2$ & 1060 \\
$15^{\mathrm{B}}$ & $3 \times 3 \times 10 / 3 \times 3 \times 10$ & $3 \times 3 / 3 \times 3$ & 809 \\
19 & $2 \times 2 \times 8 / 2 \times 2 \times 8$ & $1 \times 1 / 1 \times 1$ & 600 \\
$20^{\mathrm{C}}$ & $13.2 \times 13.2 \times 10$ & $3.3 \times 3.3 / 13.2 \times 13.2$ & 960 \\
$21^{\mathrm{C}}$ & $12 \times 12 \times 5$ & $1.5 \times 1.5 / 96 \times 96$ & 6790 \\
22 & $1.2 \times 1.2 \times 6 / 12 \times 12 \times 6$ & $3.16 \times 3.16 / 12.64 \times 12.64$ & 2500 \\
$23^{\mathrm{B}}$ & $1.5 \times 1.5 \times 10 / 39 \times 87 \times 10$ & $3.05 \times 3.05 / 36.6 \times 36.6$ & 2112 \\
\hline${ }^{\mathrm{A}}$ Ref. use LSO as scintillator crystal, the rest of the references used LYSO. \\
${ }^{\mathrm{B}}$ Measurements performed with low jitter reference detector (180 ps FWHM) and \\
extrapolated for two identical detectors. \\
${ }^{\mathrm{C}}$ Monolithic crystal used.
\end{tabular}

determination in most PET systems. In [15] many other parameters that influence CRT were studied by means of SiPMs and LYSO crystals. In order to establish the technological limits, a test with a temperature-controlled environment, optimizing the discriminator, voltage bias and other parameters using a single SiPM was performed. CRT measurements were extrapolated for a pair of detectors, and a value of 809 ps FWHM was obtained. In [19] and [20] the authors obtained a CRT of $600 \mathrm{ps}$ and $960 \mathrm{ps}$ FWHM, respectively. Both used a reference detector with a very low jitter error and LYSO crystals in combination with SiPM arrays.

There is one work in which a 64 SiPM matrix was used [21]. Again, a reference detector based on traditional PMTs and a $\mathrm{BaF}_{2}$ crystal was configured to work in combination with the array and a continuous LYSO scintillator with different crystal treatments. For a black painted crystal, a 6.79 ns FWHM CRT was achieved for both (SiPM and PMT) in coincidence. Recent publications such as [22] and [23] also use SiPM matrices. The aim of the first work consists in demonstrating the ability to resolve time and energy resolution with an array of $4 \times 4$ SiPMs coupled to pixelated LYSO crystals. For this case, the CRT was of about $2.5 \mathrm{~ns}$ FWHM. In the second case, a CRT of $2.1 \mathrm{~ns}$ was achieved with a reference low-jitter detector and a matrix of $12 \times 12$ SiPM detector pairs.

Although several tests have been successfully performed by the authors with single SiPMs coupled to individual Teflon wrapped crystal pixels [14], the combination of multiple SiPMs is not an easy task since they present a range of gains and optimum voltages that can significantly vary from one to another. In this paper, CRT studies with arrays of $12 \times 12 \mathrm{SiPMs}$ and an active area of $3 \mathrm{~mm} \times 3 \mathrm{~mm}$ each, were successfully carried out. There are also studies with arrays of comparable dimension but they refer to digital SiPMs [24][25]. Modifications of different parameters to enhance the CRT such as increasing photodetection efficiency (PDE) and signal to noise ratio through the SiPM overvoltage, as well as an optimization of the digitizer thresholds used for triggering at values of few incoming photons are considered in this work. This optimization has been shown [15] to improve the CRT by a factor of almost 3 when they are tuned together (when referring to Teflon wrapped crystals). The conditioning circuits have been improved to preserve the quality of the signal. Furthermore, CRT values when SiPMs are under low and controlled temperature conditions will be presented. A detailed study on the influence of the described parameters, including CRT, with two arrays of 144 SiPMs and LYSO crystals, will be shown and discussed. The obtained CRT values are significantly improved compared to other literature works presented above.

\section{TOF-TRIGGER ARCHITECTURE}

A trigger unit based on a TDC-FPGA [16] is in charge of receiving the signals coming from the detectors in order to determine whether a true coincidence occurred and, if so, "trigger" the PET acquisition system to digitize the required positioning signals. Constant Fraction Discriminators (CFDs) were used as digitizers in the set-up. They receive the analog signal coming from the detectors and generate a digital pulse when predefined conditions are matched. They are used to avoid dependence of the timing results on the detector voltage. The proposed trigger system also includes an embedded processor which enables the communication with the peripherals and which incorporates the possibility of implementing a coincidence map.

In this contribution, two channels for CRT measurements have been used and, thus, only two CFD digitizers were required. Values of timestamps are routed to the processor, which performs the time differences and sends the data through serial port to a PC workstation. A Labview interface collects the data and shapes a histogram from which the data are fitted to a Gaussian distribution.

\section{Characterization}

There are two main blocks that have to be carefully optimized in order to achieve the optimum CRT. On the one hand, all the components that involve the detectors such as the crystal scintillator, the SiPM dimensions and the read-out electronics, must be analyzed. On the other hand, the aspects related to the trigger system such as the TDC and digitizers should be considered. In this section, the details of the photo detectors as well as the TDC considerations are described.

\section{A. Photodetectors}

Arrays of $12 \times 12 \mathrm{SiPMs}$ have been assembled following a modular structure (see Fig. 1). These arrays are built with SensL SiPMs (mod. Micro-FB 30035), whose maximum peak 

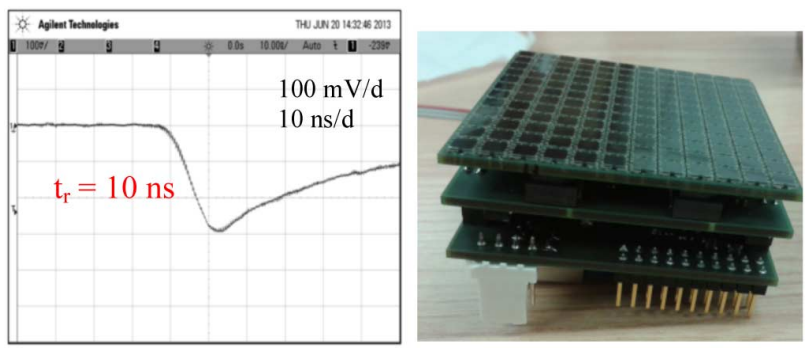

Fig. 1. Left, SiPM signal output after the conditioning stage. Right, picture of the $12 \times 12$ array of SiPMs.

efficiency is at $420 \mathrm{~nm}$. The active area is $3 \mathrm{~mm} \times 3 \mathrm{~mm}$ withmicrocells of $35 \mu \mathrm{m} \times 35 \mu \mathrm{m}$. The breakdown voltage ofthese sensors is about $25 \mathrm{~V}$. Their temperature dependence is $20 \mathrm{mV} / \mathrm{C}$. The arrays cover an area of $50.2 \mathrm{~mm} \times 50.2 \mathrm{~mm}$ with $57 \%$ of active sensitive area.

The 144 SiPM signals are connected to 3 application specific integrated circuits (ASICs), which permit up to 8 matrices of coefficients to be uploaded to estimate the photon impact position within the crystal volume [26]. Each ASIC controls 48 signals, being capable to manage up to 64 . The designed ASIC can be connected to SiPM devices whose terminal capacitance is below $40 \mathrm{pF}$. In addition to the standard output $(\approx 700 \mathrm{pF})$, these SensL SiPMs provide an additional output (named FAST) with a terminal capacitance of only $30 \mathrm{pF}$. However, due to the positive signal polarity, it was not possible to directly use this signal with the ASIC since it would not match the negative input requirement of this particular device. According to the ASIC capacitance requirements, the so-called FAST output of $12 \mathrm{pF}$ was connected to ground. In order to limit the current flowing through the new implementation, an additional series resistor was mounted with a resistance value of $10 \mathrm{k} \Omega$. Thus, the final terminal capacitance of the anode-cathode signal is $30 \mathrm{pF}$, properly matching the ASIC requirements. The ASICs provided six output signals in these experiments. One signal provided the trigger-energy, four served to reconstruct the planar impact position and one more is used to code the photon depth of interaction (DOI) within the crystal volume. In our case, this DOI encoding signal is useful only when working with monolithic crystals, and it is based on the estimation of the width of the light distribution which is closely related to the DOI [26]. The trigger-energy signal was the one considered for the present timing experiments. After the ASIC, two conditioning stages composed of two current feedback operational amplifiers take place in order to invert and amplify the signals. At the end of this process, the resulting signal has a rise time of about $10 \mathrm{~ns}\left(\mathrm{t}_{r}\right)$, as shown in Fig. 1. However, if the ASIC capacitance had been compatible, the use of the FAST output would have allowed to obtain a signal witha rise time as fast as $1 \mathrm{~ns}$ [27].

\section{B. $T D C$}

A TDC based on a delay line principle has been implemented on a Kintex-7 device (mod. xc7k325t-2ffg900). The TDC was tested previously and a time resolution below $80 \mathrm{ps} \mathrm{FWHM} \mathrm{was}$ obtained for a pair of channels [14]. Different pulses coming from the detector stage were introduced on each FPGA-TDC channel. The signals were propagated along the delay lines and a timestamp was generated for each channel. These values were sent to an embedded processor (Xilinx MicroBlaze), which computed the time differences.

The main reason of using the FPGA-TDC has been to obtain good SiPM+LYSO timing resolution in a manner that scales well to an interesting number of channels.

\section{Tests AND Results}

In the tests, different crystal topologies have been used. Pixelated crystal arrays are being used in many experiments due to their enhancement, compared to monolithic configurations, in terms of the amount of the scintillation light transferred to the photosensor. In contrast to these types of scintillators, monolithic crystals are very attractive from the point of view of preserving the light distribution when painted black, in addition to being cost effective. Since the scintillation light is isotropically spread, such designs tend to transfer a reduced amount of light to individual photosensors when compared to pixelated crystal arrays where most of the generated light is focused onto very few SiPMs. It should be mentioned that monolithic crystals with reflective walls suggest innovative methods to reconstruct the photon impact position within the crystal [28]. These tasks are outside the scope of this study, which is focused on time resolution optimization.

Fig. 2 illustrates the set-up used for the tests. Two identical SiPM arrays were used, but the scintillation crystals coupled to them varied depending on the test. They were placed in a temperature-controlled environment. The detectors were put on top of a cold-plate whose temperature was controlled through water supplied from a chiller. A temperature loss of about $10^{\circ} \mathrm{C}$ was observed between the chiller temperature and that measured at the detector. The cold-plate and detectors were placed in a porexpan box in order to keep a stable temperature. Two small ventilator fans were directed to the detector blocks in order to improve their thermal stabilization. A Radioactive ${ }^{22} \mathrm{Na}$ source $(\sim 1 \mu \mathrm{Ci})$ was placed at different positions in the line connecting the two detectors and the CRT was determined for each configuration. Signals coming from the detectors were digitized by means of CFDs (Ortec, Mod. 584) and analyzed with the developed TDC-FPGA, using two channels. In order to select the coincidence events, besides CFD thresholds, a $10 \mathrm{~ns}$ time window has been established so that the TDC discards the events having time difference outside the selected time window. Three different scenarios are presented in the following subsections.

\section{A. Scenario 1}

In this section the SiPM arrays were coupled to two pixelated arrays. One array was made out of $24 \times 24$ LYSO elements of $2 \mathrm{~mm} \times 2 \mathrm{~mm}$ size each and $10 \mathrm{~mm}$ thick, covering the whole SiPM photo sensor area, i.e. $50 \mathrm{~mm} \times 50 \mathrm{~mm}$. The second array has $27 \times 27$ LYSO elements of $1.4 \mathrm{~mm} \times 1.4 \mathrm{~mm}, 10 \mathrm{~mm}$ thick covering most of the active area. Fig. 3 shows a photograph of the experimental set-up, consisting of a SiPM array coupled to a LYSO pixelated scintillator array module placed inside the temperature-controlled box.

The source was placed at four different positions in order to calibrate the timestamp of the FPGA-TDC. Temperature was controlled at $28^{\circ} \mathrm{C}$ and voltage bias for both sensors was fixed 


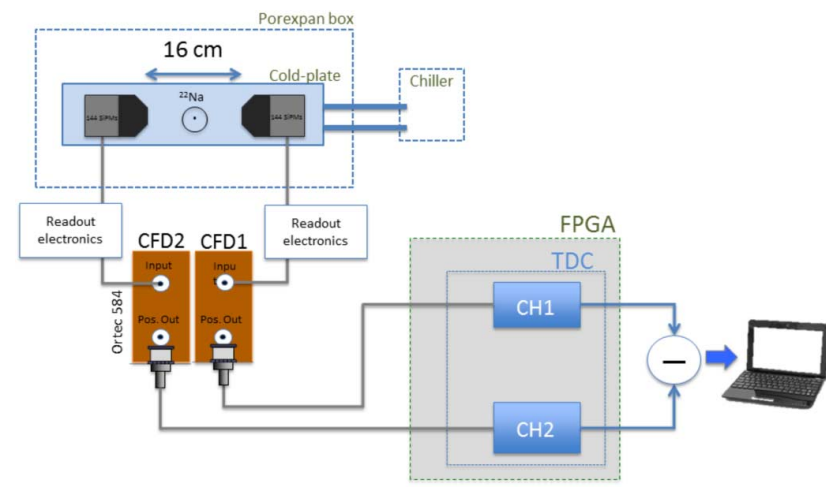

Fig. 2. Set-up used for the tests described in the paper.

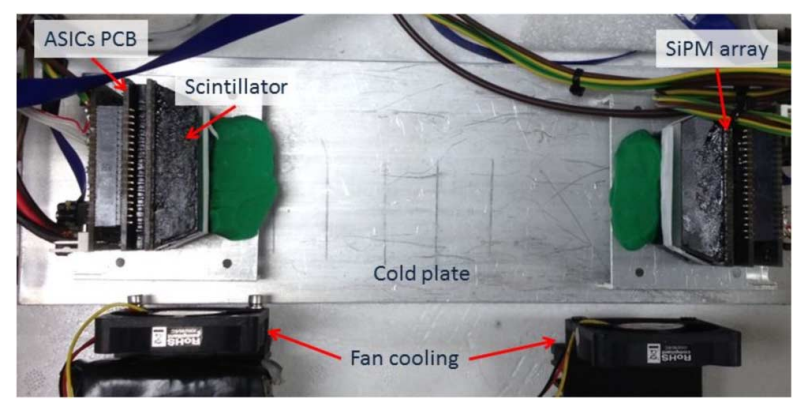

Fig. 3. Picture of the set-up showing one detector block.

at $30 \mathrm{~V}$. These environmental conditions do not affect calibration because the TDC raw count is not influenced by such parameters. Data were fitted to a regression line as shown in the upper left of Fig. 4. Both SiPM arrays were subjected to several optimizations. The effect of bias voltage, CFD thresholds and detector assembly temperature were accurately determined. Regarding the CFD threshold, a 2D contour plot shows the convenience of using certain threshold values (Fig. 4).

Values of CFD voltage threshold were trimmed in order to achieve the best detector performance and an external delay cable of $10 \mathrm{~ns}$ for both CFDs was chosen based on the $10 \mathrm{~ns}$ rise time obtained. As it has been studied by the authors in other publications such as [14] or [29], a breakeven point has to be encountered to accomplish two premises: a sufficiently low threshold value in order to take advantage of the first arrival photons (best time performance) but high enough to avoid the electronic noise, which is present in such a low level signal amplitude. The results of such a scan are shown in Fig. 4 lower left. The best CRT values were found for thresholds of $400 \mathrm{mV}$ and $300 \mathrm{mV}$, corresponding to detector array 1 and 2, respectively.

The SiPM bias voltage is directly related to the PDE. However, there exists a certain value for which PDE remains almost constant. Moreover, incrementing the bias voltage has direct impact on the dark count rate, which exponentially increases [15]. A bias voltage scan in a temperature-controlled environment of $28^{\circ} \mathrm{C}$ was carried out from $27 \mathrm{~V}$ to $33 \mathrm{~V}$, resulting in a CRT below 900 ps FWHM for $30 \mathrm{~V}_{\text {bias }}$, see the upper-right plot in Fig. 4.

The SiPM arrays were placed inside the temperature stabilized thermal box. The temperature was measured with an
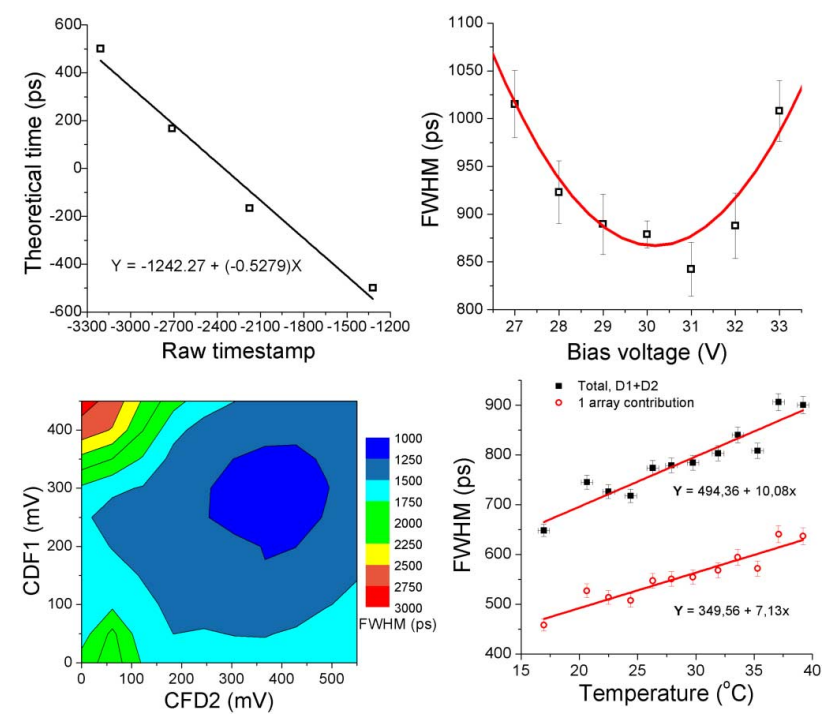

Fig. 4. TDC timestamp calibration (upper left). Optimization of bias voltage (upper right), digitizer thresholds (lower left) and temperature dependency (lower right).

on-chip sensor fastened to the SiPM array. The graph in Fig. 4 (lower right) shows the measured CRT for different temperature values. The black squares depict the CRT results for the whole system, i.e. both detectors in coincidence (D1 + D2), whereas the red circles refer to a single detector contribution, extrapolating its effect, $(\mathrm{D} 1+\mathrm{D} 2) / \sqrt{2}$. In what follows, extrapolated data concept is used to infer the expected CRT values that would be obtained for a given detector if the "other" detector were ideal, i.e. with a transit time spread $=0$. In this way we can estimate the single detector contribution to CRT. We have assumed that the contribution to the final CRT of the two LYSO arrays is almost the same, despite the fact that the dimensions of the pixels are different (1.4 and $2.0 \mathrm{~mm}$ ).

Once CRT behavior for varying bias voltage and temperature was established, temperature was stabilized at $28^{\circ} \mathrm{C}$, whereas bias voltage was set to $30 \mathrm{~V}$. This temperature provides a good time resolution without the risk of water condensation inside the set-up. CRT measurements were again considered at four different source positions, at steps of $5 \mathrm{~cm}$, and $\sim 1,700$ events were taken. Fig. 5 depicts the data measured together with Gaussian fit. The results provided CRT values from 784 ps to 1,171 ps FWHM.

A certain disparity in the CRT values of Fig. 5 can be seen. Such disparities are likely due to the scintillator dimensions, which are not the same for both detectors. This may be caused by some of the gamma rays that are not registered by the detector with less scintillator surface, causing a widening in the Gaussian distribution. Since in one module the scintillator does not cover the whole detection area, some of the gamma pairs are not properly detected (one module registers the event but the other does not), and the ratio of valid coincidences versus the random ones decreases, thus degrading the Gaussian distribution.

\section{B. Scenario 2}

In the second set of data, SiPMs were coupled to $12 \mathrm{~mm}$ thick monolithic LYSO crystals. In these experiments, different 


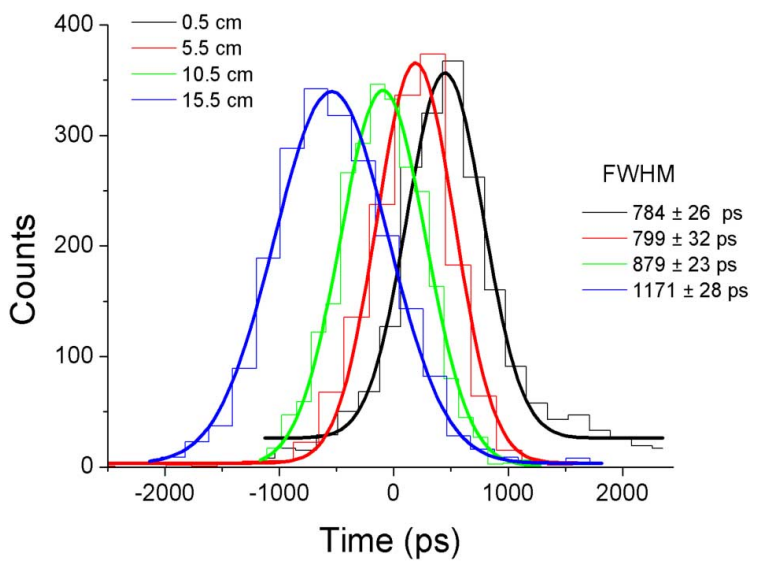

Fig. 5. CRT results for two LYSO crystal arrays at four distinct source positions. The origin of the reference corresponds to the array with pixels of $1.4 \mathrm{~mm} \times 1.4 \mathrm{~mm}$.

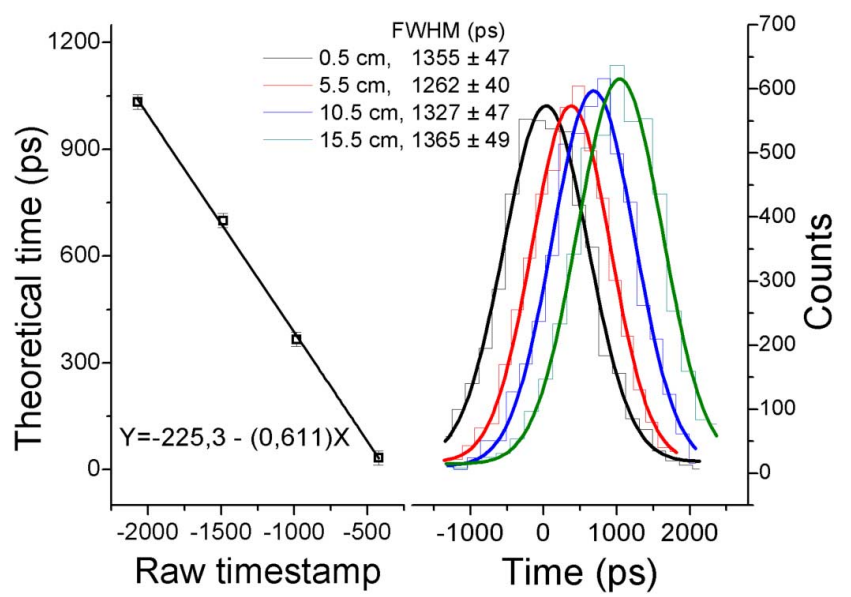

Fig. 6. CRT results for 144 SiPMs arrays and LYSO monolithic crystals when the source was sequentially moved in $5 \mathrm{~cm}$ steps.

monolithic crystal configurations were adopted in order to explore the effect of the amount of light on the CRT. Crystal blocks with trapezoidal shape have been used for both detectors. These slabs have a $40 \mathrm{~mm} \times 40 \mathrm{~mm}$ incoming radiation face and $50 \mathrm{~mm} \times 50 \mathrm{~mm}$ (12 $\mathrm{mm}$ thick) for the opposite face coupled to the SiPM array through optical grease (Saint Gobain BC-630; $\mathrm{n}=1.465)$.

Four different configurations have been implemented to carry out CRT measurements. A black-painted monolithic block is fixed on one of the detectors so that it serves as a reference detector. The monolithic block of the opposite detector was also black-paint treated in one case. In another case the lateral and entrance surfaces were wrapped with white Teflon. In the third case the entrance face was covered with a enhanced specular reflector (ESR, 3M) film, with the lateral sides black-painted. In the last test all surfaces were covered with the ESR film.

The source was first placed at four distinct positions to calibrate the timestamps provided by the TDC. In this test, the two black-painted crystals were used. The four points were fitted to a regression line. Fig. 6 shows the plot and the regression curve together with the CRTs that were found for these four positions.

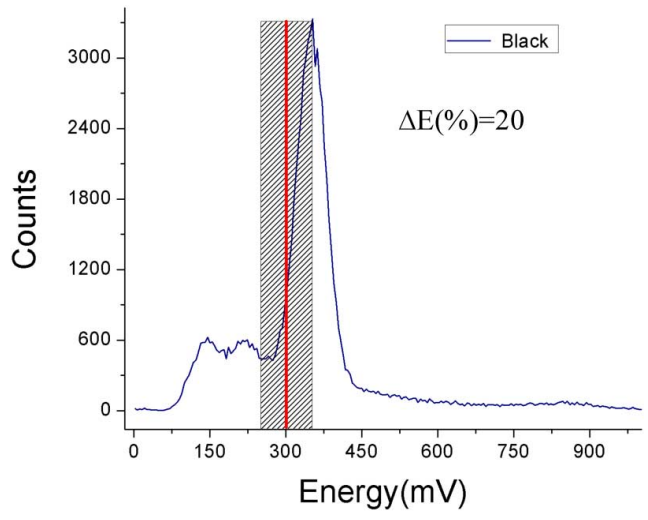

Fig. 7. Energy spectrum for the monolithic black-painted scintillator SiPM array module. The optimal values have been found in the range of $300 \mathrm{mV}$, having an energy resolution of $20 \%$.

The CRT resulted in values varying from 1.26 to $1.36 \mathrm{~ns}$ for 4,000 events. Different parameters were controlled in order to test the system performance with these crystals. Regarding the CFD threshold, optimum values were found to be $100 \mathrm{mV}$ in one detector and $150 \mathrm{mV}$ for the other. The difference in the CFD thresholds was due to a slight difference in amplification of the two SiPM trigger channels, as in Scenario 1.

The CFDs were optimized to return the best CRT through several iterations in all the following configurations. These values were fixed for the data collection in each particular scenario. Furthermore, the energy spectrum of each signal was evaluated. The selected thresholds we found are at the low energy tail of the $511 \mathrm{keV}$ photopeak. In Fig. 7, an example of the energy spectrum corresponding to the monolithic black-painted scintillator is shown, with an energy resolution close to $20 \%$. For other scintillator configurations, the optimal values were also set before the photopeak. In the case of the monolithic covered with ESR film, a resolution as good as $17 \%$ was measured.

The bias voltage was set to $30 \mathrm{~V}, 5.5 \mathrm{~V}$ overvoltage. This decision was also based on the best CRT across several voltage biases. Temperature was maintained at $15-17^{\circ} \mathrm{C}$ for all measurements, with this value being a standard working temperature.

Measurements were taken to optimize performance, as stated above, both for the CFDs threshold and for the bias voltage as well. Again, temperature effects were analyzed, as in previous scenario, and the results showed a linear tendency of $<10 \mathrm{ps} /{ }^{\circ} \mathrm{C}$ in a $10-30^{\circ} \mathrm{C}$ temperature range.

Fig. 8 shows the CRT values for the described crystal configurations. The best performance is reached with the ESR configuration (the whole crystal is covered by the film), where $1.05 \mathrm{~ns}$ CRT is achieved for both detectors in coincidence. Slightly higher CRT values of $1.07 \mathrm{~ns}$, for the white-painted crystal and black-ESR, were found. The worst CRT was determined for the case where the two black-painted slabs were used, obtaining a value of $1.35 \mathrm{~ns}$.

These results are, most likely, caused by the reduction of the amount of light transferred to the SiPM. The transit time spread is inversely proportional to the detected light, also known as jitter, of the detector. In other words, the higher the amount of light, the lower the jitter [30]. 


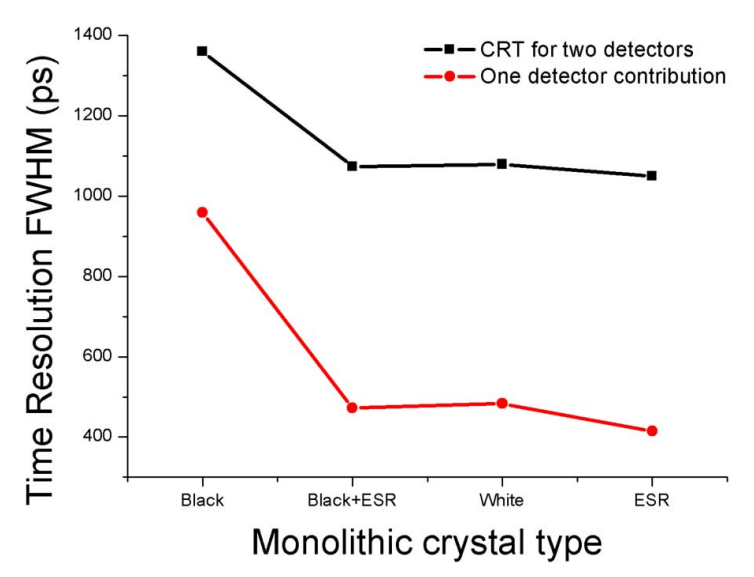

Fig. 8. CRT results for different monolithic crystal configurations in one detector and black-painted in the opposite (reference). The line with squares represents CRT values for both detectors and the line with the circles the extrapolated effect for one detector.
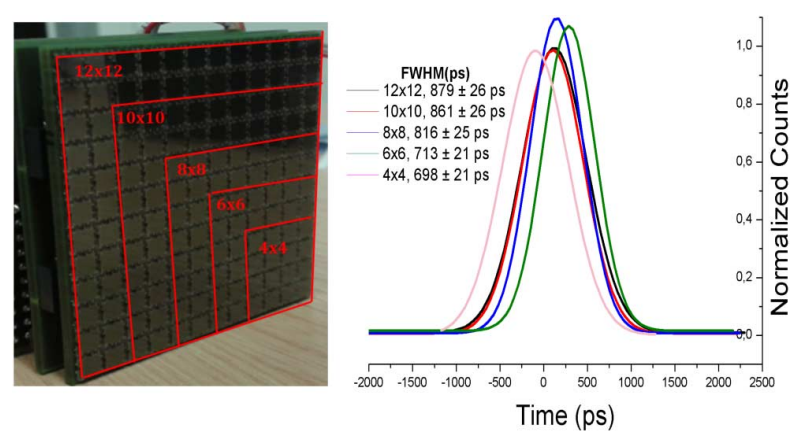

Fig. 9. CRT results for a fixed $12 \times 12$ SiPM array module and varying the amount of enabled SiPMs for the other.

It should be remarked that the results from these two scenarios come from a 144 SiPM matrix, which means that each individual SiPM is introducing a certain amount of noise into the final trigger-energy signal, since there are not individual SiPM thresholds. In the next scenario, several of the SiPMs that formed the $12 \times 12 \mathrm{SiPM}$ array will not be considered in the trigger-energy signal in order to explore its effect on the CRT.

\section{Scenario 3}

With the purpose of analyzing the SiPMs' contribution of noise to the CRT, the area of one of the SiPM arrays was selectively reduced, while maintaining the operation of the $12 \times 12$ SiPMs in the opposite detector block. The same two modules as in Scenario 1 were used. The optimum parameters found in the previous tests were selected. The number and position of the SiPMs contributing to the trigger-energy signal can be programmed via the ASICs, in charge of reading out the SiPM arrays. The source was fixed at the center of both detectors and CRT measurements were taken for 5 different matrix configurations, as shown in Fig. 9.

As expected, the results exhibit a clear improvement of the CRT with the pixel area reduction. Despite the fact that the reduction has been performed for one detector, the individual contribution of the trimmed one has been extrapolated following the quadratic relationship:

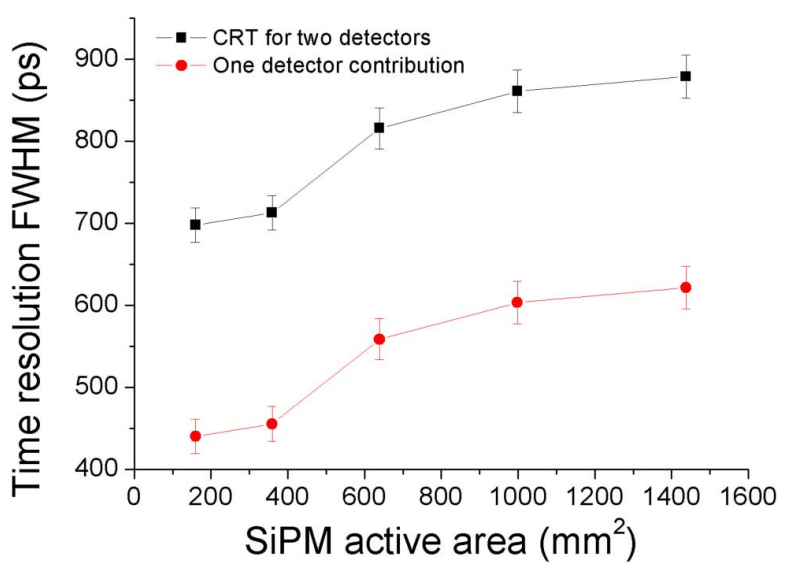

Fig. 10. Measured $\mathrm{CRT}_{\left(d_{1}+d^{2} 2\right)}$ as a function of the active area of one SiPM photo sensor array (black squares). The extrapolated contribution of the single detector with a "virtually" reduced SiPM active area $\left(\mathrm{CRT}_{(d 1)}\right)$ is shown with red circles.

$$
C R T_{d 1}=\sqrt{C R T_{\left(d_{1}+d_{2}\right)}^{2}-C R T_{d_{2}}^{2}}
$$

where $d_{1}$ is the detector with the matrix modifications, $d_{2}$ is the detector with the $12 \times 12$ fixed matrix, $C R T_{d 1(d 2)}$ is the CRT value for the detector $d_{1}\left(d_{2}\right)$ and $\left.C R T_{(} d 1+d 2\right)$ is the measured CRT obtained for the whole system, i.e. both detectors in coincidence (D1 + D2). $C R T_{d 2}$ is assumed to be the CRT value for the detector $d_{2}$ extrapolated from the measurements carried out for the full (unchanged) $d_{1}$ matrix. The measured $C R T(d 1+d 2)$ and the extrapolated contribution of the single detector with a "virtually" reduced SiPM active area $\left(C R T_{d 1}\right)$ according to equation (1), are shown in Fig. 10.

A significant CRT improvement was achieved by reducing the photosensor active area. With all the photosensors enabled $(12 \times 12$ matrix for both modules) CRT values were nearly 850 ps FWHM and, by means of reducing the SiPM array active area down to $200 \mathrm{~mm}^{2}$ (which means an array of $4 \times 4 \mathrm{SiPM}$ ), a 700 ps FWHM CRT was achieved. This value could be compared with that obtained in [20], where a $4 \times 4 \mathrm{SiPM}$ array $\left(2.85 \mathrm{~mm}^{2}\right)$ coupled to a LYSO $(1.3 \mathrm{~mm} \times 1.3 \mathrm{~mm} \times 10 \mathrm{~mm})$ was used, obtaining a CRT of $1.4 \mathrm{~ns}$.

Knowing the contribution to the CRT of the fixed $12 \times 12$ SiPM matrix detector, as from equation (1), it is possible to extrapolate its effect to obtain the CRT for one detector contribution. For the equivalent of $4 \times 4$ array pixels, the calculated CRT is about $300 \mathrm{ps}$, as the graph of Fig. 9 exhibits. This fact quantitatively demonstrates how the SiPM array assembly has a direct impact on the CRT.

\section{CONCLusions}

A large array of $12 \times 12$ SiPMs has been optimized for both monolithic and pixelated LYSO crystals. Two sensor modules have been built with a number of SiPMs that make the system suitable to explore its use in gamma ray detectors, especially in MR compatible PET systems. Several cases under different conditions for a 144 SiPM array and the designed TDC have been presented in order to explore CRT values, with the aim of achieving the best detector performance. 
The test carried out with pixelated crystals resulted in a CRT as small as 800 ps FWHM for the entire $12 \times 12 \mathrm{SiPM}$ photosensor array, measured at a sensor temperature of $28^{\circ} \mathrm{C}$, whereas a CRT of 1,260 ps FWHM has been obtained with a black painted monolithic crystal, due to the reduction of the amount of light transferred to the photosensor.

In order to explore the light effects on the system performance, different monolithic-crystal configurations have been mounted, analyzing the CRT values for each. The best performance has been achieved with the ESR film, by covering the whole detector. The results when all surfaces were black painted worsened to $\approx 1.4 \mathrm{~ns}$. These experiments corroborated the CRT improvement with the amount of light received by the photosensors.

In addition to crystal type experiments, the CFD thresholds used for digitizing purposes were optimized, as well as the bias voltage and the sensor block temperature. The best bias voltage values were obtained at $\approx 5 \mathrm{~V}$ overvoltage and, as expected, the lower the temperature the better the CRT. A deviation of $10 \mathrm{ps} /{ }^{\circ} \mathrm{C}$ in the system resolution was determined for both the pixelated and the monolithic crystal.

The two differences in time calibration slopes observed in Fig. 4 and Fig. 6 is being investigated in order to determine its possible sources. There were some conditions that changed from one experiment to another, but they need to be carefully analyzed to establish a reliable conclusion.

Finally, a 700 ps FWHM CRT (about 300 ps for one single detector) was reached when a reduced number of SiPMs were enabled, demonstrating CRT improvement with the reduction of SiPM active area and, therefore, the contribution of dark noise. It should be pointed out that the CRT values obtained with the $12 \times 12$ SiPM array are significantly better than those values reported so far in the literature for smaller $(4 \times 4$ and $2 \times 2)$ SiPM arrays [17][22] and for the similar array dimensions [23].

\section{REFERENCES}

[1] M. Conti, "State of the art and challenges of time-of-flight PET," Phys. Med., vol. 25, pp. 1-11, 2008

[2] [Online]. Available: www.healthcare.philips.com/

[3] J. Kyung-chan, "TDC module for ToF" in Proc. IEEE NSS Conf., 2007.

[4] A. Mohammad et al., "A multihit time-to-digital converter architecture on FPGA,” IEEE Trans. Instrum. Meas., vol. 58, no. 3, pp. 530-540, 2009.

[5] J. Wu, "Several key issues on implementing delay line based TDCs using FPGAs," IEEE Trans. Nucl. Sci., vol. 57, no. 3, pp. 1543-1548, Apr. 2010

[6] J. Torres et al., "Time to digital converter based on FPGA with multiple channel capability," IEEE Trans. Nucl. Sci., vol. 61, no. 1, pp. 107-114, Feb. 2013.

[7] A. Del Guerra et al., "Silicon photomultipliers (SiPMs) as novel photodetectors for PET," NucL. Instrum. Meth. Phys. Res. A, vol. 648, pp. S232-S235, 2011.

[8] N. Efthimiou et al., "Initial results on SiPMs performance for use in medical imaging," Meas. Sci. Technol., vol. 22, p. N114001, 2011.
[9] T. Kato et al., "A novel gamma-ray detector with submillimeter resolutions using monolithic MPPC array with pixelized Ce: LYSO and Ge: GGAG crystals," Nucl. Instrum. Meth. Phys. Res. A, vol. 699, pp. 235-241, 2011.

[10] H. S. Yoon et al., "Initial results of simultaneous PET/MRI experiments with an MRI-compatible Silicon Photomultiplier PET scanner," J. Nucl. Med., vol. 53, no. 4, pp. 608-614, 2012.

[11] S. Mandai1 and E. Charbon, "A $4 \times 4 \times 416$ digital SiPM array with 192 TDCs for multiple high-resolution timestamp acquisition," J. Instrum. , vol. 8, p. P05024, 2013, 10.1088/1748-0221/8/05/P05024.

[12] T. Szcze et al., "Time resolution of scintillation detectors based on SiPM in comparison to photomultipliers," in Proc. IEEE Nucl. Sci. Symp., 2010, pp. 1728-1735.

[13] F. Powolny et al., "Time based read-out of a SiPM for time of flight position emission tomography," IEEE Trans. Nucl. Sci., vol. 58, no. 3, pp. 597-604, Jun. 2011

[14] A. Aguilar et al., "Time of flight measurements based on FPGA and SiPM for PET-MR," Nucl. Instrum. Meth. Phys. Res. A [Online]. Available: http://dx.doi.org/10.1016/j nima.2013.09.008i, 2013

[15] C. L. Kim, G. C. Wang, and S. Dolinsky, "Multi-pixel photon counters for TOF PET detector and its challenges," IEEE Trans. Nucl. Sci., vol. 56, no. 3, pp. 2580-2585, Aug. 2009.

[16] G. Llosá et al., "Silicon photomultipliers and SiPM matrices as photodetectors in nuclear medicine," in Proc. IEEE Nucl. Sci. Symp., 2007, pp. 3220-3223.

[17] V. C. Spanoudaki et al., "Use of single photon counting detector arrays in combined PET/MR: Characterization of LYSO-SiPM detector modules and comparison with a LSO-APD," J. Instrum., p. P12002, 2007.

[18] S. J. Hong I. C. Song, and M. Ito et al. "An investigation into the use of Geiger-mode solid-state photomultipliers for simultaneous PET and MRI acquisition,” IEEE Trans. Nucl. Sci., vol. 55, no. 2, pp. 882-888, Apr. 2008.

[19] R. Vinke et al., "Optimizing the timing resolution of SiPM sensors for use in TOF-PET detectors," Nucl. Instrum. Meth. Phys. Res. A, vol. 610, pp. 188-191, 2009.

[20] D. Schaart et al., "A novel, SiPM-array-based, monolithic scintillator detector for PET," Phys. Med. Biol, vol. 54, pp. 3501-3512, 2009.

[21] G. Llosa et al., "Characterization of a PET detector head based on continuous LYSO cristals and monolithic, 64-pixel silicon photomultipliers matrices," Phys. Med. Biol., vol. 55, pp. 7299-7315, 2010.

[22] C. J. Thompson et al., "Measurement of energy and timing resolution of very highly pixelated LYSO crystal blocks with multiplexed SiPM readout for use in a small animal PET/MR insert," in Proc. IEEE Nucl. Sci. Symp., 2013.

[23] R. R. Raylman, "A large area, silicon photomultiplier-based PET detector module," Nucl. Instrum. Meth. Phys. Res. A, vol. 735, pp. 602-609, 2014

[24] [Online]. Available: www.research.philips.com/initiatives/digitalphotoncounting/

[25] Y. Haemisch et al., "Fully digital arrays of silicon photomultipliers (dSiPM)-a scalable alternative to vacuum photomultiplier tubes (PMT)," in Phys Proc., 2012, vol. 37, pp. 1546-1560.

[26] V. Herrero et al., "PESIC: An integrated front-end for PET applications," IEEE Trans. Nucl. Sci., vol. 55, no. 1, pp. 27-33, Feb. 2008

[27] S. Dolinsky, G. Fu, and A. Ivan, Timing resolution performance comparison for fast and standard outputs of SensL SiPMs, 2013 [Online]. Available: http://www.sensl.com/downloads/irp/2013_Dolinsky_Timing_Resolution_Performance_Comparison for Fast and Standard Outputs of SensL_SiPM.pdf

[28] H. T. Van Dam et al., "Sub-200 ps CRT in monolithic scintillator PET detectors using digital SiPM arrays and maximum likelihood interaction time estimation," Phys. Med. Biol., vol. 58, pp. 3243-57, 2013.

[29] S. Seifert et al., "The lower bound on the timing resolution of scintillation detectors," Phys. Med. Biol., vol. 57, pp. 1797-1814, 2012.

[30] F. Matthew W and E. Charbon, "System tradeoffs in gamma-ray detection utilizing SPAD arrays and scintillators," IEEE Trans. Nucl. Sci. vol. 57, no. 5, pp. 2549-2557, 2010. 